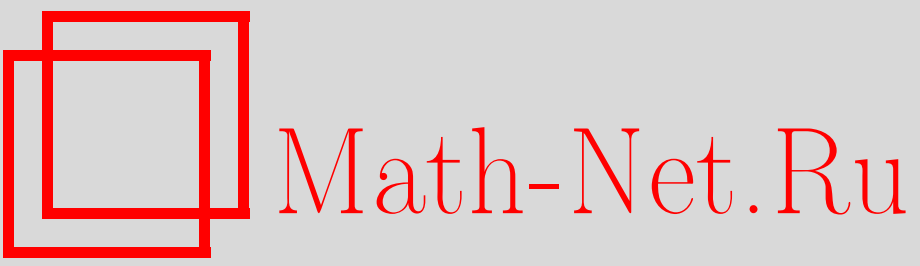

В. А. Воблый, Асимптотическое перечисление помеченных последовательнопараллельных тетрациклических графов, Итоги науки и техн. Сер. Соврем. мат. и ее прил. Темат. обз., 2020, том 187, 31-35

DOI: https://doi.org/10.36535/0233-6723-2020-187-31-35

Использование Общероссийского математического портала Math-Net.Ru подразумевает, что вы прочитали и согласны с пользовательским соглашением

http: //www. mathnet.ru/rus/agreement

Параметры загрузки:

IP: 18.209 .158 .208

26 апреля 2023 г., 02:54:35 


\title{
АСИМПТОТИЧЕСКОЕ ПЕРЕЧИСЛЕНИЕ ПОМЕЧЕННЫХ ПОСЛЕДОВАТЕЛЬНО-ПАРАЛЛЕЛЬНЫХ ТЕТРАЦИКЛИЧЕСКИХ ГРАФОВ
}

\author{
(c) 2020 г. $\quad$ В. А. ВОБЛЫЙ
}

\begin{abstract}
АннотАция. Последовательно-параллельный граф-это граф, не содержащий полный граф с четырьмя вершинами в качестве минора. Найдена асимптотика для числа помеченных связных последовательно-параллельных тетрациклических графов с большим числом вершин. Доказано, что при равномерном распределении вероятностей вероятность того, что помеченный связный тетрациклический граф является последовательно-параллельным графом, асимптотически равна $141 / 221$.
\end{abstract}

Ключевъе слова: перечисление, помеченный граф, последовательно-параллельный граф, асимптотика, вероятность.

\section{ASYMPTOTICAL ENUMERATION OF LABELED SERIES-PARALLEL TETRACYCLIC GRAPHS}

\author{
(c) 2020 V. A. VOBLYI
}

\begin{abstract}
A series-parallel graph is a graph that does not contain a complete graph with four vertices as a minor. We find an asymptotics for the number of labeled connected series-parallel tetracyclic graphs with a large number of vertices. We prove that under a uniform probability distribution, the probability of the fact that a labeled connected tetracyclic graph is a series-parallel graph is asymptotically equal to $141 / 221$.
\end{abstract}

Keywords and phrases: enumeration, labeled graph, series-parallel graph, asymptotics, probability. AMS Subject Classification: 05C30

\section{1. Введение.}

Определение 1. Граф называется последовательно-параллельным, если он не содержит полный граф с четырьмя вершинами в качестве минора (см. [9]).

Определение 2. Цикломатическим числом связного графа называется увеличенная на единицу разность между числом ребер графа и числом его вершин. Граф с цикломатическим числом, равным $k$, называется $k$-ииклическим графом.

Определение 3. Класс графов называется блочно-устойчивым, если граф принадлежит этому классу тогда и только тогда, когда каждый блок графа принадлежит этому классу (см. [10]).

Последовательно-параллельные графы используются при построении надежных коммуникационных сетей (см. [11]). 
В [9] найдена асимптотика для чисел помеченных связных и 2-связных последовательно-параллельных графов с большим количеством вершин. В [3] перечислены помеченные последовательно-параллельные 2-связные графы по числу вершин. Число помеченных последовательнопараллельных трициклических и тетрациклических 2-связных графов с заданным числом вершин найдено в [6] и [5], соответственно. В [4] перечислены по числу вершин помеченные связные последовательно-параллельные трициклические графы.

В статье найдена асимптотика для числа помеченных связных последовательно-параллельных тетрациклических графов с большим числом вершин. Доказано, что при равномерном распределении вероятностей вероятность того, что помеченный связный тетрациклический граф является последовательно-параллельным графом, асимптотически равна 141/221. Также доказано, что при равномерном распределении вероятностей вероятность того, что помеченный связный последовательно-параллельный тетрациклический граф является кактусом, асимптотически равна $7 / 47$.

2. Перечисление графов. Рассматриваются неориентированные простые связные графы.

Теорема 1. Для числа $S P(n, 4)$ помеченных связных последовательно-параллельных тетраииклических графов с $n$ вершинами при $n \geqslant 6$ верна формула

$$
\begin{gathered}
S P(n, 4)=\frac{(n-1) !}{24}\left[z^{n-1}\right] e^{n z}\left(\frac{n^{3} z^{8}}{16(1-z)^{4}}+\frac{n^{2} z^{4}\left(12 z^{3}-13 z^{4}+4 z^{5}\right)}{4(1-z)^{6}}+\right. \\
+\frac{n\left(12 z^{3}-13 z^{4}+4 z^{5}\right)^{2}}{12(1-z)^{8}}+\frac{n z^{2}\left(70 z^{4}-127 z^{5}+98 z^{6}-38 z^{7}+6 z^{8}\right)}{2(1-z)^{8}}+ \\
\left.+\frac{48 z^{11}-450 z^{10}+1860 z^{9}-4415 z^{8}+6520 z^{7}-5813 z^{6}+2430 z^{5}}{10(1-z)^{10}}\right) .
\end{gathered}
$$

Доказательство. Обозначим через $B(n, k)$ число помеченных $k$-циклических блоков с $n$ вершинами, через $B_{k}(z)$ - экспоненциальную производящую функция для $B(n, k), S(n, k)$ - число помеченных связных $k$-циклических графов с $n$ вершинами. В [1] получено выражение

$$
S(n, k)=\frac{(n-1) !}{n k !}\left[z^{-1}\right] e^{n z} Y_{k}\left(n 1 ! B_{1}^{\prime}(z), n 2 ! B_{2}^{\prime}(z), \ldots, n k ! B_{k}^{\prime}(z)\right) z^{-n},
$$

где $\left[z^{-1}\right]$ - оператор формального вычета (см. [7]), а $Y_{k}\left(x_{1}, \ldots, x_{k}\right)$ - многочлены разбиений (многочлены Белла). Для этих многочленов известно выражение (см. [8, с. 173])

$$
Y_{k}\left(x_{1}, \ldots, x_{k}\right)=\sum_{\pi(k)} \frac{k !}{m_{1} ! \ldots m_{k} !}\left(\frac{x_{1}}{1 !}\right)^{m_{1}} \ldots\left(\frac{x_{k}}{k !}\right)^{m_{k}},
$$

где суммирование проводится по всем разбиениям $\pi(k)$ числа $k$, т.е. по всем неотрицательным решениям $\left(m_{1}, m_{2}, \ldots, m_{k}\right)$ уравнения $m_{1}+2 m_{2}+\cdots+k m_{k}=k, m_{i} \geqslant 0, i=1, \ldots, k$.

Формула (2) верна не только для всего класса связных графов, но и для блочно-устойчивого его подкласса (см. [3]). Известно, что класс последовательно-параллельных графов является блочноустойчивым классом графов (см. [10]). Поэтому заменим в $(2) S(n, k)$ на $S P(n, 4)$ и будем считать, что функции $B_{k}(z)$ относятся к классу помеченных последовательно-параллельных графов.

Так как

$$
Y_{4}\left(x_{1}, x_{2}, x_{3}, x_{4}\right)=x_{1}^{4}+6 x_{1}^{2} x_{2}+3 x_{2}^{2}+4 x_{1} x_{3}+x_{4}, \quad x_{i}=n i ! B_{i}^{\prime}(z)
$$

(см. $[8$, c. 246]), то имеем

$$
\begin{aligned}
S P(n, 4)=\frac{(n-1) !}{24 n}\left[z^{-1}\right] e^{n z}\left(n^{4}\left(B_{1}^{\prime}(z)\right)^{4}+\right. & 12 n^{3}\left(B_{1}^{\prime}(z)\right)^{2} B_{2}^{\prime}(z)+ \\
& \left.+12 n^{2}\left(B_{2}^{\prime}(z)\right)^{2}+24 n B_{1}^{\prime}(z) B_{3}^{\prime}(z)+24 n B_{4}^{\prime}(z)\right) z^{-n} .
\end{aligned}
$$


Унициклический блок - это простой цикл (последовательно-параллельный граф), поэтому $B(n, 1)=(n-1) / 2$. Все бициклические блоки являются последовательно-параллельными графами, и в [12], [6], [5] соответственно найдены формулы

$$
\begin{aligned}
& B(n, 2)=\frac{n !(n-3)(n+2)}{24}, \\
& B(n, 3)=\frac{n !(n-3)(n-4)}{5760}\left(3 n^{3}+36 n^{2}+71 n+50\right), \\
& B(n, 4)=\frac{n !}{80640}\left(n^{5}+30 n^{4}+257 n^{3}+768 n^{2}+960 n+504\right)\left(\begin{array}{c}
n-3 \\
3
\end{array}\right) .
\end{aligned}
$$

Таким образом, имеем

$$
\begin{gathered}
B_{1}(z)=\sum_{n=3}^{\infty} \frac{1}{2}(n-1) ! \frac{z^{n}}{n !}, \quad B_{1}^{\prime}(z)=\frac{z^{2}}{2(1-z)}, \quad B_{2}(z)=\frac{z^{4}(3-2 z)}{12(1-z)^{3}}, \quad B_{2}^{\prime}(z)=\frac{12 z^{3}-13 z^{4}+4 z^{5}}{12(1-z)^{4}}, \\
B_{3}(z)=\frac{z^{5}\left(28-47 z+28 z^{2}-6 z^{3}\right)}{48(1-z)^{6}}, \\
B_{4}(z)=\frac{405 z^{6}-1004 z^{7}+1066 z^{8}-609 z^{9}+186 z^{10}-24 z^{11}}{240(1-z)^{9}} \\
B_{4}^{\prime}(z)=\frac{48 z^{11}-450 z^{10}+1860 z^{9}-4415 z^{8}+6520 z^{7}-5813 z^{6}+2430 z^{5}}{240(1-z)^{10}}
\end{gathered}
$$

Суммирование рядов для $B_{2}(z), B_{3}(z), B_{4}(z)$, а также дифференцирование было выполнено с помощью пакета программ Maple.

Подставляя в (3) выражения для $B_{1}^{\prime}(z), B_{2}^{\prime}(z), B_{3}^{\prime}(z), B_{4}^{\prime}(z)$, получим формулу (1).

В следующей таблице представлены числа $S P(n, 4)$, вычисленные с помощью теоремы 1 и пакета программ Maple.

\begin{tabular}{|c|c|c|c|c|c|c|}
\hline$n$ & 6 & 7 & 8 & 9 & 10 & 11 \\
\hline$S P(n, 4)$ & 1215 & 116361 & 7614936 & 435101373 & 23642892000 & 1270611137565 \\
\hline
\end{tabular}

\section{3. Асимптотика и вероятность.}

Лемма 1. Введем обозначения

$$
p(z, q)=\sum_{i=0}^{q} c_{i} z^{i}, \quad A_{n}(m, q)=\left[z^{-1}\right] \frac{p(z, q) e^{n z} z^{-n}}{(1-z)^{m}} ;
$$

тогда при фиксированных $m, q u n \rightarrow \infty$ верна асимптотика

$$
A_{n}(m, q) \sim \frac{\sqrt{\pi} p(1, q) n^{n+m / 2}}{n ! 2^{m / 2} \Gamma((m+1) / 2)} .
$$

Доказательство. Пусть $U(a, b, z)$ - вырожденная гипергеометрическая функция Трикоми. В [4] найдено разложение

$$
\frac{e^{n z}}{(1-z)^{m}}=\sum_{p=0}^{\infty} \frac{n^{p+m}}{p !} U(m, m+p+1, n) z^{p},
$$

а в [2] при фиксированных числах $a$ и $m$ и $n \rightarrow \infty$ получена асимптотика

$$
U(a, n-m, n) \sim \frac{\sqrt{\pi}}{(2 n)^{a / 2} \Gamma\left(\frac{a+1}{2}\right)} .
$$


Учитывая, что $n ! /(n-k) ! \sim n^{k}$ при фиксированном $k$ и $n \rightarrow \infty$, получим

$$
\begin{aligned}
A_{n}(m, q)=\left[z^{-1}\right] \sum_{p=0}^{\infty} & \sum_{i=0}^{q} \frac{n^{p+m}}{p !} c_{i} z^{p+i-n} U(m, m+p+1, n)=\sum_{i=0}^{q} \frac{c_{i} n^{n+m-i-1}}{(n-i-1) !} U(m, n+m-i, n) \sim \\
& \sim \sum_{i=0}^{q} \frac{n !}{(n-i-1) !} \frac{c_{i} n^{n+m-i-1}}{n !} \frac{\sqrt{\pi}}{(2 n)^{m / 2} \Gamma\left(\frac{m+1}{2}\right)} \sim \frac{n^{n+m}}{n !} \frac{\sqrt{\pi}}{(2 n)^{m / 2} \Gamma\left(\frac{m+1}{2}\right)} \sum_{i=0}^{q} c_{i},
\end{aligned}
$$

что равносильно (4).

Теорема 2. Для числа $S P(n, 4)$ помеченных связных последовательно-параллельных тетрациклических графов с $n$ вершинами при $n \rightarrow \infty$ имеет место асимптотика

$$
S P(n, 4) \sim \frac{47}{8064} n^{n+4} .
$$

Доказательство. Введем обозначения

$$
\begin{gathered}
p_{1}(z, 8)=z^{8}, \quad p_{1}(1,8)=1 ; \quad p_{2}(z, 9)=12 z^{7}-13 z^{8}+4 z^{9}, \quad p_{2}(1,9)=3 ; \\
p_{3}(z, 10)=\left(12 z^{3}-13 z^{4}+4 z^{5}\right)^{2}, \quad p_{3}(1,10)=9 ; \\
p_{4}(z, 10)=70 z^{6}-127 z^{7}+98 z^{8}-38 z^{9}+6 z^{10}, \quad p_{4}(1,10)=9 ; \\
p_{5}(z, 11)=48 z^{11}-450 z^{10}+1860 z^{9}-4415 z^{8}+6520 z^{7}-5813 z^{6}+2430 z^{5}, \quad p_{5}(1,11)=180 .
\end{gathered}
$$

Из выражения (1) с помощью леммы получим

$$
\begin{array}{r}
S P(n, 4)=\frac{(n-1) !}{24}\left(\frac{n^{3}}{16} A_{n}(4,8)+\frac{n^{2}}{4} A_{n}(6,9)+\frac{n}{12} A_{n}(8,10)+\frac{n}{2} A_{n}(8,10)+\frac{1}{10} A_{n}(10,11)\right) \sim \\
\begin{array}{r}
\sim \frac{(n-1) !}{24}\left(\frac{\sqrt{\pi} p_{1}(1,8) n^{n+5}}{16 n ! 2^{2} \Gamma(5 / 2)}+\frac{\sqrt{\pi} p_{2}(1,9) n^{n+5}}{4 n ! 2^{3} \Gamma(7 / 2)}+\frac{\sqrt{\pi} p_{3}(1,10) n^{n+5}}{12 n ! 2^{4} \Gamma(9 / 2)}+\right. \\
\left.+\frac{\sqrt{\pi} p_{4}(1,10) n^{n+5}}{2 n ! 2^{4} \Gamma(9 / 2)}+\frac{\sqrt{\pi} p_{5}(1,10) n^{n+5}}{10 n ! 2^{5} \Gamma(11 / 2)}\right) \sim \\
\sim \frac{n^{n+4}}{24}\left(\frac{1}{48}+\frac{1}{20}+\frac{1}{140}+\frac{3}{70}+\frac{2}{105}\right)=\frac{47}{8064} n^{n+4} .
\end{array}
\end{array}
$$

Зададим на множестве помеченных связных тетрациклических графов с $n$ вершинами равномерное распределение вероятностей.

Следствие 1. При $n \rightarrow \infty$ вероятность $P_{n}$ того, что помеченный тетрациклический граф с $n$ вершинами является последовательно-параллельным графом, асимптотически равна 141/221.

Доказательство. Доказательство. Пусть $f(n, n+3)$-число помеченных связных графов с $n$ и $n+3$ ребрами (тетрациклических графов). В [13] Райт нашел следующую асимптотику при $n \rightarrow \infty$ :

$$
\begin{gathered}
f(n, n+k) \sim f_{k} n^{n+(3 k-1) / 2}, \quad f_{k}=\frac{\sqrt{\pi} 3^{k}(k-1) ! d_{k}}{2^{(3 k-1) / 2} \Gamma((3 k / 2))}, \quad k \geqslant 1 ; \\
d_{1}=d_{2}=\frac{5}{16}, \quad d_{k+1}=d_{k}+\sum_{s=1}^{k-1} \frac{d_{s} d_{k-s}}{(k+1)\left(\begin{array}{l}
k \\
s
\end{array}\right)}, \quad k \geqslant 2 .
\end{gathered}
$$

Поэтому имеем

и, следовательно,

$$
d_{3}=d_{2}+\frac{1}{6} d_{1}^{2}=\frac{1105}{7776}, \quad f_{3}=\frac{54 \sqrt{\pi} d_{3}}{128 \Gamma(9 / 2)}=\frac{9}{140} d_{3}, \quad f(n, n+3) \sim \frac{221}{24192} n^{n+4}
$$

$$
P_{n}=\frac{S P(n, 4)}{f(n, n+3)} \sim \frac{141}{221} \quad \text { при } n \rightarrow \infty \text {. }
$$


Следствие 2. При $n \rightarrow \infty$ вероятность $\bar{P}_{n}$ того, что помеченный последовательно-параллельный тетрачиклический граф с п вериинами является кактусом, асимптотически равна $7 / 47$.

Доказательство. Пусть $C a(n, k)$ - число помеченных $k$-циклических кактусов с $n$ вершинами. В [1] найдена следующая асимптотика при $n \rightarrow \infty:$

$$
C a(n, k) \sim \frac{\sqrt{\pi}}{2^{3 k / 2} k ! \Gamma((k+1) / 2)} n^{n+3 k / 2-2} .
$$

Поэтому при $n \rightarrow \infty$ имеем

$$
\bar{P}_{n}=\frac{C a(n, 4)}{S P(n, 4)} \sim \frac{\sqrt{\pi} n^{n+4} 8064}{2^{6} \cdot 24 \cdot \Gamma(5 / 2) \cdot 47 \cdot n^{n+4}}=\frac{7}{47} .
$$

\section{СПИСОК ЛИТЕРАТУРЫ}

1. Воблый В. А. О перечислении помеченных связных графов с заданными числами вершин и ребер// Дискр. анал. иссл. опер. - 2016. - 23, № 2. - С. 5-20.

2. Воблый В. А. Число помеченных внешнепланарных графов $k$-циклических графов// Мат. заметки. 2018. - 103, № 5. - С. 657-666.

3. Воблый В. А. Второе соотношение Риддела и следствия из него// Дискр. анал. иссл. опер. - 2019. 26, № 1. - C. 20-32.

4. Воблый В. А. Перечисление помеченных последовательно-параллельных трициклических графов// Итоги науки техн. Сер. Совр. мат. прилож. Темат. обзоры. - 2020. - 177. - С. 132-136.

5. Воблый В. А. Число помеченных последовательно-параллельных тетрациклических блоков// Прикл. дискр. мат. -2020 . - № 47. - С. 57-61.

6. Воблый B. А., Мелешко А. М. О числе помеченных последовательно-параллельных трициклических блоков// Мат. XV Междунар. конф. «Алгебра, теория чисел и дискретная геометрия. Современные проблемы и приложения» (Тула, 28-31 мая 2018 г.). - Тула: ТПГУ. - С. 168-170.

7. Гульден Я., Джексон Д. Перечислительная комбинаторика. - М.: Наука, 1990.

8. Риордан Дж. Комбинаторные тождества. - М.: Наука, 1982.

9. Bodirsky M., Gimenez O., Kang M., Noy M. Enumeration and limit laws of series-parallel graphs// Eur. J. Combin. - 2007. - 28, № 8. - P. 2091-2105.

10. McDiarmid C., Scott A. A random graphs from a block stable class// Eur. J. Combin. — 2016. — 58. — P. 96-106.

11. Radhavan S. Low-connectivity network design on series-parallel graphs// Networks. — 2004. — 43, № 3 . - P. 163-176.

12. Wright E. M. The number of connected sparsely edged graphs, II// J. Graph Theory. — 1978. — 2, № 4. - P. 299-305.

13. Wright E. M. The number of connected sparsely edged graphs, III// J. Graph Theory. — 1980. — 4, № 4. - P. 393-407.

Воблый Виталий Антониевич

Всероссийский институт научной и технической информации, Москва

E-mail: vitvobl@yandex.ru 